\title{
Adaptive Antenna Technique for Mobile Communication
}

\author{
Ryszard J. Katulski \\ Technical University of Gdansk, Department of Radiocommunication \\ e-mail: rjkat@sunrise.pg.gda.pl
}

Keywords: mobile telecommunication, adaptive antenna system, direction of arrival procedures, microstrip technology.

Abstract: In this paper the adaptive array properties analysis due to its application in a base station of cellular mobile telecommunication system is presented. First, taking into account the interference problem, the base station antenna system with adaptive directional properties in horizontal plane is motivated. The functional structure of the adaptive processor is described. Next, the problem of the direction of radio signal arrival (doa) estimation have been presented. Especially, the MUSIC and ESPRIT doa algorithms are analysed. A structure of the adaptive array in microstrip technology is proposed. In conclusion, the usefulness of the adaptive technique in a base station of mobile cellular telecommunication is confirmed.

\section{INTRODUCTION}

The land mobile communication, especially cellular telephone networks are very modern form of telecommunication systems. Mobile telecommunication in general and cellular service in particular have been forefront of research and development activities. The progressive development of the wireless telecommunication systems, which are realized by use the radio link equipments, connects with the increaising of an interference level. The limitation of frequency band and the interference problem are important questions connected with cellular mobile systems, particularly in the future high capacity systems. A solution of the problems can be obtain by 
application the frequency reuse and cell splitting as well as cell sectorization with directional form of an antenna radiation pattern. These require to use for base station of the mobile network special antenna system, especially an adaptive antenna array technique with single narrow beams dynamically assign to iluminate the mobile units [1].

The researches and development activities connected with mobile communication systems, including the adaptive antenna theory and technique, have been done in Radiocommunication Department of the Technical University of Gdansk, Poland [2].

\section{INTERFERENCE PROBLEM IN MOBILE RADIO SYSTEM}

The main element of each mobile wireless system is the radio link consists of a base station and mobile unit, between which a radiowave propagation medium is existed. The antenna equipments installed in both base and mobile unit are interfaces between the wire and wireless part of the radio link, which structure in a short functionally form is presented in Figure 1.

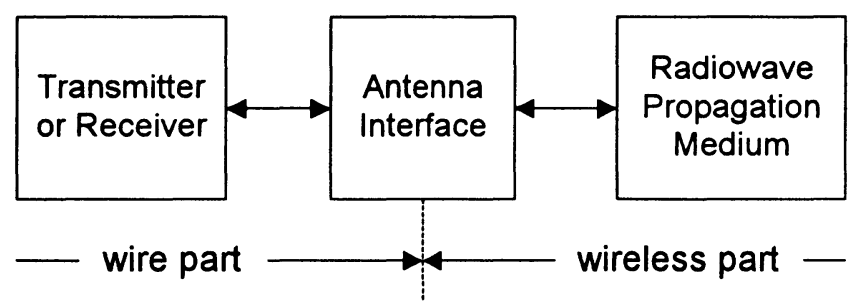

Figure 1. Functionally form of a radio link structure

Properties of these antennas influence on the radio link operation condition, especially the shape of these antennas radiation patterns, decide about the values of the interference signals. Each transmitter equipment is a source of the interference signals, which space distribution depends on the transmitter antenna directivity properties. Moreover, the directivity properties of an antenna operated with a receiver equipment influence on the received interference signals. Therefore, the interference signal level depends on a shape of the antenna radiation pattern [3]

Taking into account the movements of the mobile units the simple antenna form with omnidirectional radiation pattern is prefered to apply in a base station and mobile units. But, the directivity properties of the simple antenna structure are not optimal, especially for the base station of the high capacity cellular networks. The intereference problem requires to use in the base station the directional antenna with multi-beam radiation pattern for multi- 
channels operation, in which each beam iluminates the individual mobile unit. This requirement can be realized by adaptive antenna system with mobile beams. For example, if the probability ratio $p_{\text {one-beam }}\left(s_{w} \leq s_{i}+p_{r}\right)$ at mobile unit, for omnidirectional radiation pattern in a base station, is assumed as:

$$
p_{\text {one-beam }}\left(s_{w} \leq s_{i}+p_{r}\right)=p
$$

where: $\quad s_{w} \quad-\quad$ value of the wanted signal,

$s_{i} \quad-\quad$ value of the co-channel interference signal,

$p_{r} \quad, \quad$ protection ratio,

the probability ratio $p_{\text {multi-beam }}^{\prime}\left(s_{w} \leq s_{i}+p_{r}\right)$ at mobile unit, for mutli-beam radiation pattern in base station, is resulted as:

$$
p_{\text {one-beam }}\left(s_{w} \leq s_{i}+p_{r}\right)=\frac{p}{m},
$$

where $m$ is number of beams.

In result, the directional antennas are convenient to use in the base stations of a cellular telecommunication network, especially with multi-beam radiation pattern for multi-channels operation in order to obtain the better value of the spectrum utilization efficiency, i.e. number of channels per megahertz per square kilometer [4].

\section{ADAPTIVE PROCESSOR}

An adaptive antenna system is the antenna array that controls its own radiation pattern, by means of feedback control loop, while an adaptive processor operates. Adaptive antenna systems have been employed in military technique. Application in civil communication is not widespread, although some potential schemes have been suggested $[5,6]$.

A short form of the adaptive processor system is presented in Fig. 2, here the set $\{x$ of radio signals received by each antenna elements is multiplicated by set $\{w\}$ of complex weights, and next are summed to produce an output signal. The output signal $y$ is compared with reference signal $r$ and in result the error signal $e$ is obtained to steering with set $\{x$ the processor unit [7].

The dynamic form of the output signal can be presented in matrix notice as:

where

$$
y(t)=\mathbf{w}^{\mathrm{T}} \cdot \mathbf{x},
$$

$$
\mathbf{x}=\mathbf{s}+\mathbf{n},
$$


in which $\mathbf{X}$ and $\mathbf{n}$ are respectively: the desire signals matrix and noise with interference signals matrix.

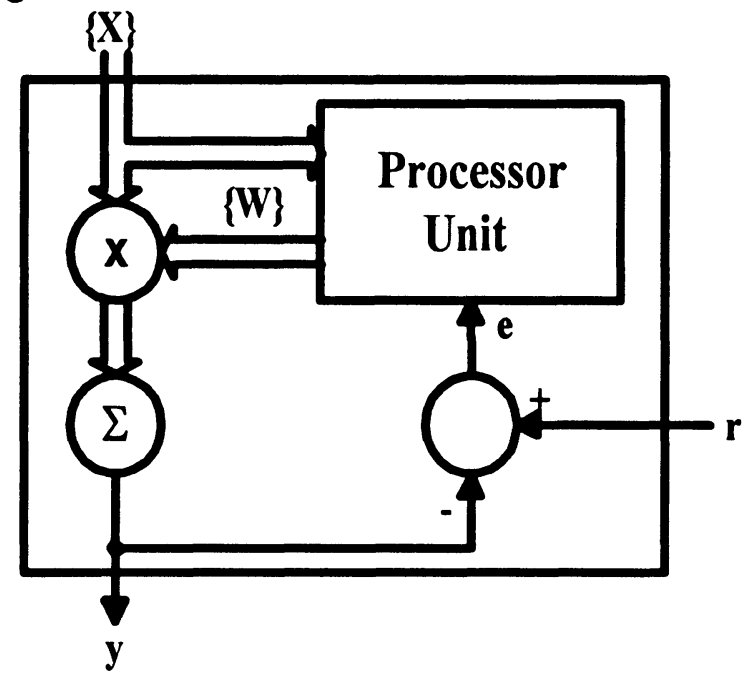

Figure 2. The adaptive processor structure

The difference between the output signal and the reference signal is the error signal:

$$
e(j)=r(j)-\mathbf{w}(j)^{T} \cdot \mathbf{x}(j),
$$

which should be minimaized by finding optimal values of the weights set, i.e.:

$$
\mathbf{w}_{\text {optimla }}=\Phi^{-1}(x, x) \cdot \Phi(x, r)
$$

where $\Phi(x, x)$ is a matrix of the crosscorelations and autocorrelations ratios of the received input signals, and $\Phi(x, r)$ is a vector of crosscorelations ratios between the input signals and the reference signals.

The knowledge of the direction of radio signal arrival, in short form socalled doa estimation, is very important to improve the efficiency of the optimal weights finding procedure, especially taking into account the real time requirements conditions.

\section{DOA ESTIMATION PROCEDURES}

From different methods which can be used for calculation of the doa, the two procedures: MUSIC and ESPRIT are extensively tested for application in adaptive processor scheme. 
In the first step of the MUSIC method, the covariance matrix of the radio signals received by antenna array elements shoud be estimated by use the formal procedure:

$$
\hat{\mathbf{R}}_{\mathbf{x}}=\frac{1}{J} \sum_{j=1}^{J}\left[\mathbf{x}(j) \cdot \mathbf{x}(j)^{H}\right]
$$

in which the $J$ is number of the radio signals samples. Next, by eigenvectors corresponds to the largest eigenvalues of the covariance matrix so the peaks in the direction of radio signal arrival can be obtained. The eigenvectors can be classified into two groups: one spanning the signal with noise subspace and one spanning a pure noise subspace. A matrix of the eigenvectors $\mathbf{e}$ is formed which span the pure noisespace. This matrix is orthogonal to the angles $\Theta$ from which the radio signal is arriving and it is possible to obtain high peaks in these direction $\Theta$ by solving the equation:

$$
|d(\Theta)|^{2}=\sum_{i}\left|\mathbf{G}^{\mathbf{H}}(\Theta) \cdot \mathbf{e}_{i}\right|^{2}=0,
$$

in which $\mathbf{G}^{\mathbf{H}}()$ is hermitian matrix of the directivity functions of the antenna array elements [8].

Due to the ESPRIT doa procedure the two $\Delta$ spaced parallel subarrays antenna elements are applied. The scheme to obtain the angles of radio signal arrival based on the eigenproblem analysis. First, the covariance matrix of the radio signals received by the two antenna subarrays should be estimated. Next, the angles $\Theta$ can be estimated by way:

$$
\Theta=\sin ^{-1}\left\{\frac{c}{\omega \cdot \Delta} \cdot \arg (\Phi)\right\},
$$

in which vector $\Phi$ is estimated due to eigenvalues of the covariance matrix.

\section{THEORETICAL INVESTIGATIONS}

The directive properties of the adaptive antenna system with LMS optimization procedure improve by doa estimation were theoretically investigated. The modelled adaptive array was theoretically tested at 900 $\mathrm{MHz}$ common for GSM mobile communication system by use simulation system which consists of:

- PC computer for modelling of the mobile unit scenario, 
- the signal processor for doa and LMS procedures realization.

Changes in the weight vector are made along the direction of the estimated gradient vector $\nabla$ with scalar constant $k_{s}$ to control rate of convergence and stability, i.e.:

$$
\mathbf{w}(j+1)=\mathbf{w}(j)+k_{s} \cdot \nabla(j)
$$

with

$$
\nabla(j)=-2 r(j) \cdot \mathbf{x}(j)
$$

The obtained results of the influences of the adaptive process parameters on an attenuation of interference signals are exemplary presented in Figs. 3 and 4 .

The influence of number $N$ of the antenna array elements is shown in Fig. 3 . The number of the antenna elements also depends on the require directivity properties of the antenna array and on the maksimum value of the mobile unit speed.

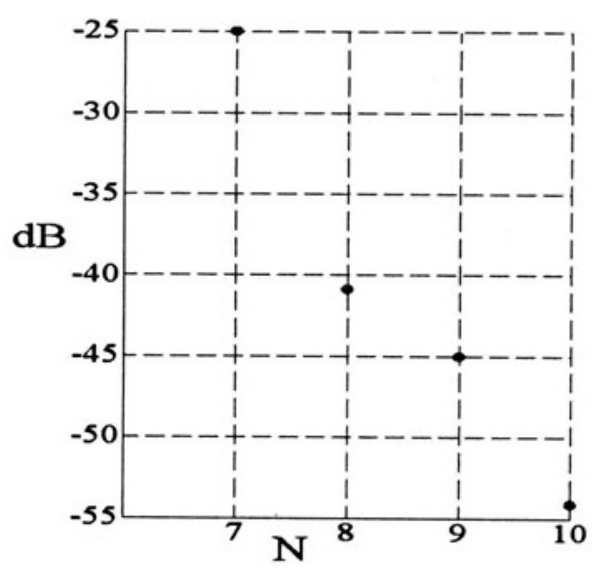

Figure 3. Interference signal attenuation vs. number of the antenna array elements

The influence of the number $N_{i}$ of iteration steps on the interference signals attenuation is presented in Fig. 4. The optimal value of iteration steps depends on requirements to acceptable value of the interference signal attenuation and of a speed of the adaptive processor unit 


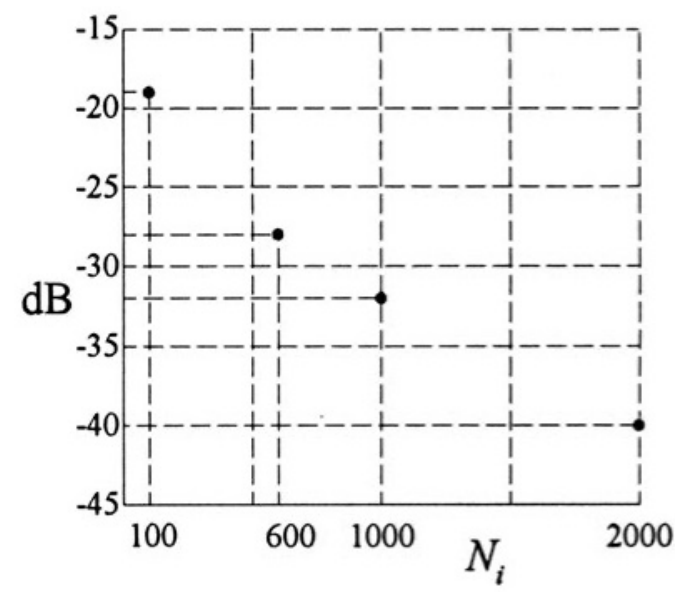

Figure 4. Interference signal attenuation vs. the number of iteration steps

Taking into account that the adaptive procedure is realized by iteration process, the maksimum time of single adaptation should be determined. The practically accepted maksimum time $t_{\max }$ depends on:

- the beamwidth $\beta$ of the antenna array radiation pattern,

- the speed $v$ of the mobile unit,

- and the distance $R$ between the mobile unit and the base station.

The relation between the time $t_{\max }$ and the above mentioned parameters can be described as:

$$
t_{\max }=\frac{\pi \cdot \beta \cdot R}{360 \cdot v}
$$

The limited values of the mobile unit speed obtained in described simulation experiments are presented in Table I. The obtained results show the requirements for quality of the adaptive signal processor, taking into account the real time scenario.

Table 1: Limited values of the mobile unit speed

\begin{tabular}{|c|c|}
\hline$R(k m)$ & $v\left(\frac{k m}{h}\right)$ \\
\hline 0,1 & 1,8 \\
\hline 0,5 & 9 \\
\hline 4 & 36 \\
\hline 6 & 72 \\
\hline
\end{tabular}


At the and, the tested doa procedures can be characterized as:

- relative long calculation time is the main fault of the MUSIC algorithm,

- taking into account the inaccuracy of the antenna elements performance, the ESPRIT procedure is better that MUSIC.

\section{CONCEPT OF THE ANTENNA SYSTEM}

The microstrip antenna technology characterized by thin compact form is very attractive to application in modem radiocommunication systems, especially in mobile telecommunication. The proposed microstrip adaptive array consists patch rectangular radiating elements with circular polarization, to reduce the depolarization effect which is observed in mobile communication. To obtain the circular polarization the special two layer form of the microstrip radiating element corner fed should be applied. By stacking two patches in a double-layer structure, the antenna bandwith can be increased and dual-frequency properties can be obtained due to the two frequency subbands adequate to down and uplink operation [9].

The conceptional short form of the proposed microstrip adaptive array system is shown in Fig. 5.

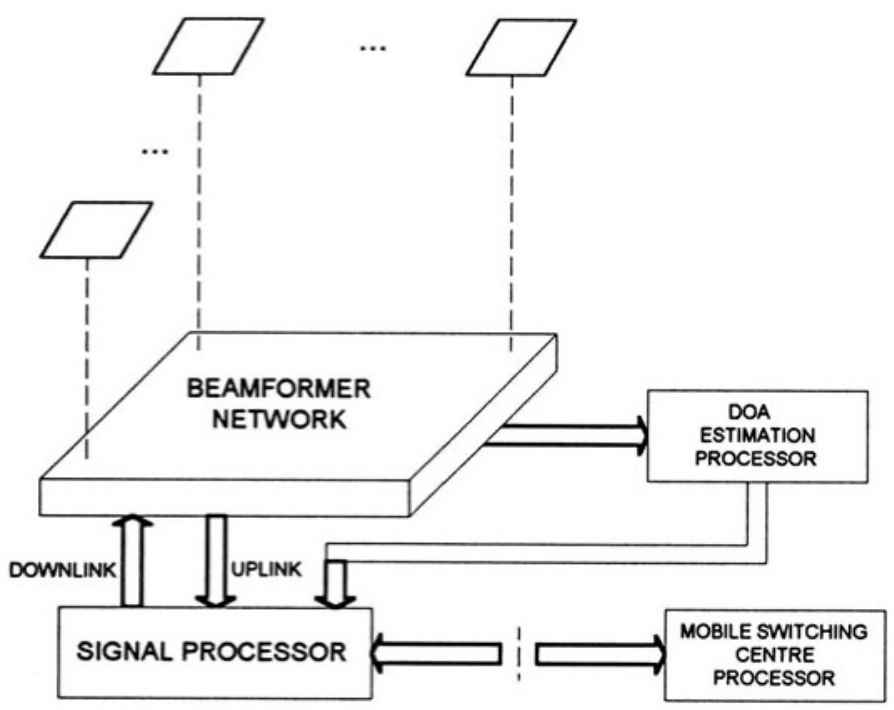

Figure 5. Concept of the microstrip adaptive array system 


\section{CONCLUSION}

The obtained results show the usefulness of the theoretically tested adaptive antenna system for application in the base station of the mobile cellular telecommunication.

\section{REFERENCES}

[1] Macario R.C.V.: Personal and Mobile Radio Systems. IEE Telecomm. Series 25, Peter Peregrinius Ltd., 1991.

[2] Katulski R.J.: Base Station Antenna System for Cellular Network. Proc. of the Int. Wiss. Colloq., pp. 904-909, Germany-Ilmenau 1992.

[3] Katulski R.J.: The EMC Antenna Aspect of Radio Systems Planning. Proc. of the Int. Symp. on Electromagnetic Compatibility; pp. 604-607, Japan-Tokyo 1999.

[4] Hammuda H.: Spectral Efficiency of Cellular Land Mobile Radio Systems. Proc. of the 38th IEEE Vehicular Technology Conf., pp. 616-622, PA-Philadelphia 1988.

[5] Hubermark S.: Smart Antennas - Intelligent Options. Mobile Europe, pp. 51-58, March 1996.

[6] Dam H.: Smart Antennas in the GSM System. TSUNAMI TestBed Demonstration Seminar, no. 18/1, A alborg University, Denmark, 1996.

[7] Compton T.: Adaptive Antennas. Prentice Hall, Inc., 1988.

[8] Katulski R.J.: Algorithm MUSIC for a Cellular Network Application, Proc. of the National Telecomm. Symp. KST-99, vol. D, pp. 117-123, Poland-Bydgoszcz 1999 (in Polish).

[9] Katulski R.J.: Application of a Stacked Microstrip Antenna for Dual-Band Operation with Circular Polarization. Proc. of the Int. Wiss. Colloq., pp. 73-77, Germany-Ilmenau 1995. 